\title{
The impact of chronic alcohol overuse on heart function and prognosis: layer-specific longitudinal strain and mid-term outcome analysis
}

\author{
Piotr Hamala', Jarosław D Kasprzak', Anna Bińkowska², Kamilla Zawitkowska-Witczak², Marlena Broncel³, \\ Anna Piekarska ${ }^{4}$, Karina Wierzbowska-Drabik \\ 11 st Department and Chair of Cardiology, Medical University of Lodz, Łódź, Poland \\ 2Department of Internal Diseases and Geriatrics, Bieganski Memorial Hospital, Łódź, Poland \\ ${ }^{3}$ Department of Internal Diseases and Clinical Pharmacology, Medical University of Lodz, Łódź, Poland \\ ${ }^{4}$ Department of Infectious Diseases and Hepatology, Medical University of Lodz, Łódź, Poland
}

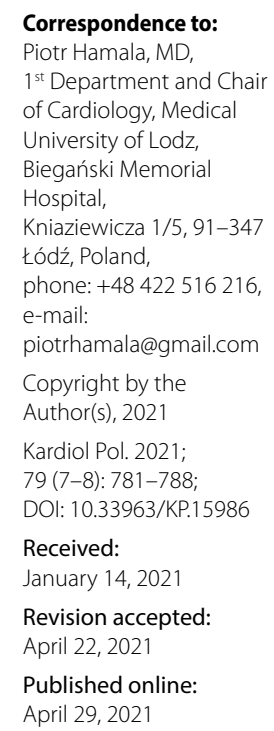

\begin{abstract}
A B STR A C T
Background: The exact effects of alcohol drinking on cardiac function are not clear.

Aims: This study aimed to determine the relationship between consumed amount of alcohol, myocardial injury, and prognosis.

Methods: Myocardial function and cardiac outcomes were examined in subjects with chronic alcoholism by classical and strain echocardiographic parameters, including global (GLS) and layer-specific longitudinal strain of the endocardial (GLSendo) and epicardial (GLSepi) layer. One group of 65 alcohol-overusers (ALC), median (IQR, interquartile range) age 44 (38-51) years, was compared with 30 controls (CG).
\end{abstract}

Results: Median (IQR) alcohol dose (in alcohol units, $1 \mathrm{AU}=1 \mathrm{~g}$ of ethanol) per week was 30 (12-51) AU in ALC and 0 in CG; $P<0.001$, and the mean (SD, standard deviation) drinking period was 16 (9) years. ALC patients demonstrated higher left ventricular (LV) mass and impaired diastolic function. The ALC group demonstrated lower median (IQR) LV ejection fraction (EF): $52 \%$ (37\%-57\%) vs 60\% (55\%-63\%) $(P<0.001)$; GLS: $17 \%(9 \%-20 \%)$ vs $19 \%(18 \%-21 \%)(P=0.01)$; absolute layer-specific strain values. GLSendo $<19 \%$ and GLSepi $<15 \%$ predicted worsened mid-term prognosis, as did elevated $\mathrm{N}$-terminal brain natriuretic peptide (NT-proBNP) and lower EF and GLS.

Conclusions: Long-term alcohol overuse, even with a rather low reported median (IQR) dose of 4 (2-7) $\mathrm{AU}$ per day resulted in LV hypertrophy, diastolic and systolic dysfunction. Diminished $G L S<18 \%$, endocardial $<19 \%$ and epicardial $<15 \%$ layer strain predicted combined endpoints but did not significantly improve the prognostic power of tested models, based on NT-proBNP and EF in follow-up.

Key words: alcohol induced cardiomyopathy, left ventricular hypertrophy, diastolic function, left atrial volume index, global longitudinal strain, layer-specific strain.

Kardiol Pol 2021; 79, 7-8: 781-788

\section{INTRODUCTION}

It was estimated by the World Health Organzation in 2018 that 2.4 billion people, i.e., $43 \%$ of the global population over 15 years old, consumed alcohol [1]. In particular, western countries have a higher incidence of dilated cardiomyopathy with an alcoholic etiology, ranging from $4 \%$ to $47 \%[2,3]$. Even so, the pathophysiology of alcohol-induced myocardial damage remains unclear, and there is a need for deeper study of the relationship between the consumed ethanol dose and severity of myocardial injury and outcomes. Novel imaging modalities provide an opportunity for more sensitive assessment of heart damage [4]. Deformation parameters with the leading global longitudinal strain allow myocardial function to be characterized quantitatively, and often more precisely than conventional variables. Furthermore, the myocardial wall can be divided into tiers according to the dominant direction of myocardial fibers (viz. endo-, epi- and midwall layers), which may be followed separately during deformation assessment [4]. 
WHAT'S NEW?

To our knowledge, this is the first study to compare the diagnostic and prognostic value of layer-specific strain with classical echocardiography parameters in chronic alcohol abusers. Our findings indicate that long-term alcohol overuse, mean (SD, standard deviation) value of 16 (9) years, with a rather low reported median (IQR, interquartile range) alcohol dose of 4 (2-7) alcohol units per day caused left ventricle hypertrophy, diastolic and systolic dysfunction with an increase in left atrium volume and the impairment of global longitudinal strain. Univariate analysis found that elevated $\mathrm{N}$-terminal brain natriuretic peptide, decreased ejection fraction and impaired global and layer longitudinal strain were related to worsened prognosis. Calculated cut-off values for global longitudinal left ventricular strain <18\%, global longitudinal epicardial strain <15\% and global longitudinal endocardial strain <19\% may have diagnostic and prognostic potential; however, they did not add any significant benefit to classical parameters in the small scale of the present study.

\section{METHODS}

\section{Patient population}

Clinical and laboratory data was collected from two groups, viz. alcohol over-users (ALC) and controls (CG) over the period 2015-2020. The alcohol dose was expressed in alcohol units $(A U)$, with one $A U$ representing ten grams of pure ethanol, irrespective of beverage type. The declared amount of alcohol consumed during a patient's life, i.e., total dose (TD), was expressed in AU, expressed as liters. Daily or weekly alcohol dose was calculated as TD divided by a period of time, i.e., AUD being alcohol units per day and AUW being alcohol units per week. The approximate accuracy of the declared alcohol dose was indirectly verified with the Michigan Alcohol Screening Test (MAST), which indicates whether the patient is addicted to alcohol and assesses addiction severity. Patients with a MAST score $<4$ (very low risk of alcohol addiction) were not included in the study.

The ALC group was formed by consecutive patients with history of alcohol overuse hospitalized in four departments of Biegański Memorial Hospital: the Department of Internal Diseases and Geriatrics, the Department of Internal Diseases and Clinical Pharmacology, the Cardiology Department, as well as the Department of Infectious Diseases and Hepatology. The study was approved by the Medical University of Lodz Ethics Committee and all patients provided written informed consent to participate in the study.

The inclusion criteria comprised the following: age $>18$ years, reported alcohol overuse $\geq 10$ AUW in any period of patient's life, recruitment to the ALC group, sinus rhythm at the time of examination. In addition, informed consent had to be given for the study and follow-up.

The exclusion criteria included any of the following: diagnosed coronary artery disease (history of myocardial infarction, revascularization), strong clinical suspicion of coronary artery disease with typical chest pain, positive for ischemia results of stress tests or scintigraphy, significant lesions in coronary angiography, persistent or permanent atrial fibrillation, diagnosed cardiomyopathies, primary significant valve diseases or pregnancy.

The CG was formed by patients without symptoms or coronary artery disease, diabetes or uncontrolled hy- pertension or any cardiomyopathies or valve diseases. All patients denied regular alcohol consumption: sporadic consumption $\leq 2 \mathrm{AU}$ per week was allowed and reported by three subjects.

\section{Echocardiographic data collection}

Echocardiographic examinations were performed with an E9 or E95 Vivid Ultrasound System (GE Healthcare, Horten, Norway) and transferred to a workstation for off-line analysis (EchoPAC, version 201, GE Healthcare). Global longitudinal strain (GLS), endocardial (GLSendo) and epicardial strain (GLSepi) were displayed as absolute values of myocardial shortening. Echocardiographic measurements were performed according to recent guidelines. The following norms were adopted: GLS $\geq 19.7 \%$, GLSendo $\geq 24.1 \%$, GLSepi $\geq 19.2 \%$ [5-9]. Strain parameters were obtained based on high-quality 2D images with 70-90 frames per second.

\section{Statistical analysis}

Data analysis was performed with MedCalc version 12.7.0.0 (MedCalc Software Ltd, Ostend, Belgium) and Excel version 14.0.7237 (Redmond, WA, USA). Variables were classified as normally or non-normally distributed with the D'Agostino-Pearson test. Quantitative variables with a normal distribution were presented as means and standard deviations (SD), and those with a non-normal distribution as medians and interquartile ranges (IQR). The results for categorical variables are presented as percentages. Qualitative data was compared using the chi-square test and the Fisher's exact test. Quantitative variables were compared using the Student's t-test for normal distributions, or the Mann-Whitney test for non-normal distributions. The correlation coefficient was calculated as the Pearson's or Spearman's coefficient for normal and non-normal distribution respectively. The combined endpoint was defined as cardiac-related hospitalization, i.e., heart failure (HF) exacerbation, myocardial infarction or ischemia or arrhythmias, or cardiac death. Survival data was analyzed with the Kaplan-Meier method and displayed as the Kaplan-Meier curve. Exact Binomial Confidence Intervals for the Area Under the Curves (AUC) in ROC analysis were calculated and, in the next step, AUC comparison was performed by DeLong method. 
Table 1. Clinical characteristics

\begin{tabular}{|c|c|c|c|}
\hline & Alcohol overuse group $(n=65)$ & Control group $(n=30)$ & P-value \\
\hline Age, years, median (IQR) & $44(38-51)$ & $40(32-55)$ & 0.39 \\
\hline Sex, men, n (\%) & 50 (77) men & $20(66)$ men & 0.52 \\
\hline $\mathrm{BMI}, \mathrm{kg} / \mathrm{m}^{2}$, median (IQR) & $25(22-27)$ & $26(22-27)$ & 0.35 \\
\hline $\mathrm{BSA}, \mathrm{m}^{2}$, mean (SD) & $1.9(0.19)$ & $1.9(0.18)$ & 0.86 \\
\hline SBP, mm Hg, mean (SD) & $121(19)$ & $123(14)$ & 0.43 \\
\hline DBP, mm Hg, mean (SD) & $76(12)$ & $74(9)$ & 0.33 \\
\hline $\mathrm{HR}$, bpm, mean (SD) & $79(12)$ & $66(13)$ & $<0.001$ \\
\hline $\mathrm{TD}, \mathrm{dm}^{3}$, median (IQR) & $201(6-34)$ & $0(0-0)$ & $<0.001$ \\
\hline AUW, median (IQR) & $30(12-51)$ & $0(0-0)$ & $<0.001$ \\
\hline \multicolumn{4}{|c|}{ Comorbidities, pharmacological therapy and symptoms } \\
\hline Arterial hypertension, n (\%) & $25(39)$ & $5(17)$ & 0.06 \\
\hline Dyslipidemia, n (\%) & $26(40)$ & $9(30)$ & 0.48 \\
\hline Diabetes, n (\%) & $7(11)$ & $2(6)$ & 0.69 \\
\hline Chronic kidney disease, n (\%) & $2(3)$ & $1(3)$ & 0.52 \\
\hline Gastritis, n (\%) & $6(9)$ & $0(0)$ & 0.17 \\
\hline $\begin{array}{l}\text { Alcoholic hepatis steatosis/inflammation, } \\
\mathrm{n}(\%)\end{array}$ & $19(29)$ & $0(0)$ & 0.002 \\
\hline Hepatic cirrhosis, n (\%) & $18(28)$ & $0(0)$ & 0.003 \\
\hline Esophageal varices, n (\%) & $5(8)$ & $0(0)$ & 0.17 \\
\hline Cholelithiasis, n (\%) & $2(3)$ & $0(0)$ & 1.0 \\
\hline COPD, n (\%) & $3(5)$ & $0(0)$ & 0.55 \\
\hline Smoking, n (\%) & $33(51)$ & $2(6)$ & 0.002 \\
\hline$\beta$-blockers, n (\%) & $50(85)$ & $6(20)$ & $<0.001$ \\
\hline Ivabradine, n (\%) & $5(8)$ & $0(0)$ & 0.17 \\
\hline ACEI/ARB, $n(\%)$ & $25(42)$ & $6(20)$ & 0.06 \\
\hline Spironolactone, n (\%) & $36(61)$ & $0(0)$ & $<0.001$ \\
\hline Diuretics, n (\%) & $33(56)$ & $0(0)$ & $<0.001$ \\
\hline Calcium antagonists, n (\%) & $3(5)$ & $2(7)$ & 0.65 \\
\hline Statins, n (\%) & $11(19)$ & $9(30)$ & 0.18 \\
\hline NYHA 0, n (\%), no symptoms & $7(11)$ & $30(100)$ & $<0.001$ \\
\hline NYHA I, n (\%) & $27(42)$ & $0(0)$ & \\
\hline NYHA II, n (\%) & $28(44)$ & $0(0)$ & \\
\hline NYHA III, n (\%) & $2(3)$ & $0(0)$ & \\
\hline NYHA IV, n (\%) & $0(0)$ & $0(0)$ & \\
\hline
\end{tabular}

Abbreviations: $\mathrm{ACEl}$, angiotensin converting enzyme inhibitors; $\mathrm{ARB}$, angiotensin receptor blockers; $\mathrm{AUW}$, alcohol units per week; $\mathrm{BMI}$, body mass index; $\mathrm{BSA}$, body surface arena; COPD, chronic obstructive pulmonary disease; DBP, diastolic blood pressure; H'R, heart rate; NYHA, New York Heart Association class; SBP, systolic blood pressure; SD, standard deviation; TD, total dose of alcohol during whole life

\section{RESULTS}

\section{Clinical and laboratory characteristics}

The ALC group consisted of 65 patients, 50 (77\%) of whom were men. The median (IQR) age was $44(38-51)$ years. CG consisted of 30 patients, 22 (66\%) of whom were men. Both groups were similar, according to age, sex, and systolic (SBP) and diastolic (DBP) blood pressure, see Table 1. The median (IQR) weekly alcohol dose (AUW) was 30 (12-51) $A U$ for ALC and $0(0-0)$ AU for CG $(P<0.001)$. The median (IQR) total alcohol dose (TD) was 201 (6-34) liters of pure ethanol in ALC vs 0 (0-0) in CG.

The mean (SD) time of alcohol consumption declared by patients was 16 (9) years. The median (IQR) time between the last alcohol ingestion and echocardiography examination, i.e., free time (FT), was 10 (7-21) days. The most popular type of drink was vodka, as well as other forms of distilled alcohol, with $26 \%$ of patients reported drinking it as a solo beverage and $87 \%$ with another type of alcoholic drink, such as beer or wine. The mean (SD) MAST score was
25 (10), which indicated a high level of alcohol addiction in the studied group.

The prevalence of arterial hypertension was similar between the groups, although more intensive hypertension therapy was observed in ALC. In addition, smoking was more prevalent in the ALC group (51\%) than the CG group (6\%) $(P=0.002$; Table 1).

In the electrocardiogram, an atrioventricular first-degree block was present in three patients, left bundle branch block in two, right bundle branch block and left anterior hemiblock in one patient each. There were no additional ST segment abnormalities.

The ALC group demonstrated a higher median N-terminal brain natriuretic peptide (NT-proBNP) level, i.e. 414 (97-1774) pg/ml, than the CG group, i.e. 44 (39-311) pg/ml ( $P<0.001$; Supplementary material, Figure S1). ALC also demonstrated higher median (IQR) corpuscular volume (MCV), i.e., $101 \mathrm{fL}$ (36-123), than the CG group, $90 \mathrm{fL}$ (83-92); $P<0.001$ and a higher serum aspartate transaminase level (AST): 64 IU/L (40-147) vs $18 \mathrm{IU} / \mathrm{L}$ (17-24) $(P=0.01$; Table 2$)$. 
Table 2. Laboratory characteristics

\begin{tabular}{|c|c|c|c|}
\hline & Alcohol group $(n=65)$ & Control group $(n=30)$ & $P$-value \\
\hline NT-proBNP, pg/ml, median (IQR) & $414(97-1774)$ & $44(39-311)$ & $<0.001$ \\
\hline $\mathrm{MCV}, \mathrm{fL}$, median (IQR) & $101(36-123)$ & $90(83-92)$ & $<0.001$ \\
\hline Creatinine, mg/dl, median (IQR) & $0.8(0.6-0.9)$ & $0.7(0.7-1.0)$ & 0.78 \\
\hline AST, IU/I, median (IQR) & $64(40-147)$ & $18(17-24)$ & 0.01 \\
\hline ALT, IU/I, median (IQR) & $42(26-86)$ & $22(17-44)$ & 0.23 \\
\hline De Ritis ratio (AST/ALT), median (IQR) & $1.4(0.85-2.55)$ & $1.2(1.0-1.8)$ & 0.75 \\
\hline
\end{tabular}

Abbreviations: ALT, alanine transaminase; AST, aspartate transaminase; MCV, mean corpuscular volume; NT-proBNP, N-terminal brain natriuretic peptide

Table 3. Echocardiography characteristics, alcohol versus control group

\begin{tabular}{|c|c|c|c|}
\hline & Alcohol group $(n=65)$ & Control group $(n=30)$ & $P$-value \\
\hline LVd, mm, mean (SD) & $48(12)$ & $48(4)$ & 0.14 \\
\hline LVs, mm, median (IQR) & $28(23-40)$ & $32(31-35)$ & 0.06 \\
\hline IVSd, mm, mean (SD) & $13(3)$ & $9(1)$ & $<0.001$ \\
\hline PWd, mm, median (IQR) & $12(11-14)$ & $10(9-11)$ & $<0.001$ \\
\hline LVMI, g/m², median (IQR) & $119(91-155)$ & $93(75-110)$ & 0.002 \\
\hline RWT, median (IQR) & $0.5(0.4-0.6)$ & $0.4(0.4-0.4)$ & 0.001 \\
\hline LA, mm, mean (SD) & $43(8)$ & $36(5)$ & 0.005 \\
\hline LAV, ml, median (IQR) & $67(49-86)$ & $40(36-49)$ & $<0.001$ \\
\hline LAVI, I/m², median (IQR) & $34(25-45)$ & $21(18-23)$ & $<0.001$ \\
\hline TAPSE, mm, median (IQR) & $22(20-26)$ & $22(21-26)$ & 0.47 \\
\hline $\mathrm{RVS}^{\prime}, \mathrm{cm} / \mathrm{s}$, mean (SD) & $12(3)$ & $13(2)$ & 0.11 \\
\hline E/A, median (IQR) & $1.1(0.8-1.2)$ & $1.3(1.1-1.4)$ & 0.01 \\
\hline $\mathrm{E}, \mathrm{m} / \mathrm{s}$, mean $(\mathrm{SD})$ & $70(19)$ & $83(18)$ & 0.03 \\
\hline E/e', median (IQR) & $7.4(5-10)$ & $6.1(5.0-6.7)$ & 0.006 \\
\hline $\mathrm{EF}, \%$, median (IQR) & $52(37-57)$ & $60(55-63)$ & $<0.001$ \\
\hline GLS, \%, median (IQR) & $17(9-20)$ & $19(18-21)$ & 0.01 \\
\hline GLSendo, \%, median (IQR) & $19(12-23)$ & $24(21-27)$ & 0.007 \\
\hline GLSepi, \%, median (IQR) & $14(9-18)$ & $19(16-20)$ & 0.007 \\
\hline $\mathrm{EF}<50 \%, \mathrm{n}(\%)$ & $25(38)$ & $0(0)$ & $<0.001$ \\
\hline LVMI, female/male $>115 / 95 \mathrm{~g} / \mathrm{m}^{2}, \mathrm{n}(\%)$ & $35(55)$ & $6(22)$ & 0.008 \\
\hline $\mathrm{LAVI}>34 \mathrm{ml} / \mathrm{m}^{2}, \mathrm{n}(\%)$ & $33(51)$ & $0(0)$ & $<0.001$ \\
\hline GLS $<19.7 \%, n(\%)$ & $45(69)$ & $10(33)$ & 0.002 \\
\hline
\end{tabular}

Abbreviations: $E / A$, wave E velocity to wave A velocity ratio; EF, left ventricular ejection fraction; GLS, global longitudinal left ventricular strain; GLSendo, global longitudinal endocardial strain; GLSepi, global longitudinal epicardial strain; IVSd, interventricular septum diastolic thickness; LA, left atrial diameter; LAV, left atrial volume; LAVI, left atrial volume index; LVd, left ventricular diastolic diameter; LVMI, left ventricular mass index; LVs, left ventricular systolic diameter; PWd, posterior wall diastolic thickness; RVS', wave $\mathrm{S}^{\prime}$ velocity; RWT, relative wall thickness; SD, standard deviation; TAPSE, tricuspid annular plane systolic excursion

\section{Echocardiography characteristics}

The ALC and CG groups demonstrated similar left ventricular diastolic and systolic diameters, but a significant difference was observed for intraventricular septum (IVS) and posterior wall thickness (PW) (Table 3). The median (IQR) left ventricular mass index (LVMI) was 119 (91-155) $\mathrm{g} / \mathrm{m}^{2}$ in ALC and $93(75-110) \mathrm{g} / \mathrm{m}^{2}$ in CG $(P=0.002$; Figure 1$)$. Systolic function, expressed as median (IQR) left ventricular (LV) ejection fraction (EF), was impaired in ALC: $52 \%$ $(37 \%-57 \%)$ vs $60 \%(55 \%-63 \%)$ in CG $(P<0.001)$, as well as a few diastolic function parameters.

The prevalence of abnormal $\mathrm{EF}$ (defined as $\mathrm{EF}<50 \%$ ) was $38 \%$ in the ALC group vs $0 \%$ in CG $(P<0.001)$. The ALC group demonstrated significant enlargements for all dimensions of the left atrium (LA): LA diameter, LA volume and volume index (LAVI), as indicated in Table 3 and Figure 1. The median (IQR) E/A ratio was higher in $\mathrm{CG}$, i.e. $1.3(1.1-1.4)$ than $A L C$, i.e. $1.1(0.8-1.2)(P=0.01)$; in addition, the mean (SD) E wave velocity was higher in CG, i.e. 83 (18) than in ALC, i.e. $70(19)(P=0.03)$; however, the median
(IQR) E/e' ratio was higher in ALC: $7.4(5-10)$ than in CG, $6.1(5.0-6.7)(P=0.006)$.

However, no significant differences were observed regarding the mean values for the classical right ventricular parameters: tricuspid annular plane systolic excursion or right ventricle $S^{\prime}$ wave velocity (Table 3 ).

The strain data was described in absolute values. The median (IQR) global longitudinal LV strain (GLS) values were $17 \%(9 \%-20 \%)$ in ALC vs $19 \%(18 \%-21 \%)$ in CG $(P=0.01)$. Both median (IQR) layer strains were lower in ALC: median (IQR) GLSendo was $19 \%$ (12\%-23\%) vs $24 \%$ $(21 \%-27 \%)(P=0.007)$ and GLSepi was $14 \%(9 \%-18 \%)$ vs $19 \%(16 \%-20 \%)(P=0.007)$, as indicated in Table 3 and Figure 2.

$\mathrm{EF}<50 \%$ was observed in $38 \%$ of ALC patients and elevated LAVI $\left(>34 \mathrm{ml} / \mathrm{m}^{2}\right)$ in $51 \%$. In contrast, none of the CG patients demonstrated any such impairments $(P<0.001)$. Deformation impairment, expressed as GLS $<19.7 \%$, was present in 45 patients (69\%) of the ALC group, and in 10 controls (33\%) $(P=0.002$; Table 3$)$. 


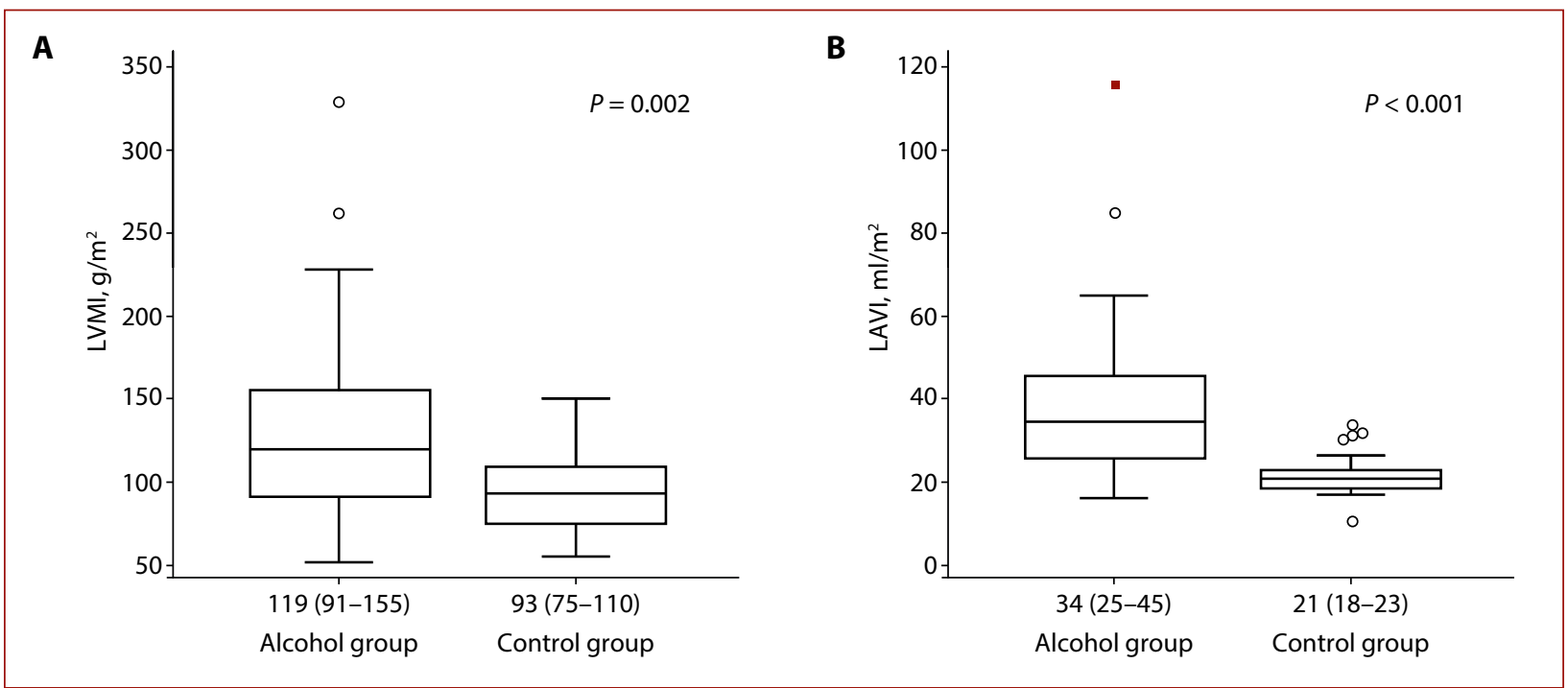

Figure 1. Increased left ventricular mass index and atrial volume index in the alcohol group as compared to the control group. A. Left ventricular mass index. B. Left atrial volume index.

Abbreviations: LAVI, left atrial volume index; LVMI, left ventricular mass index
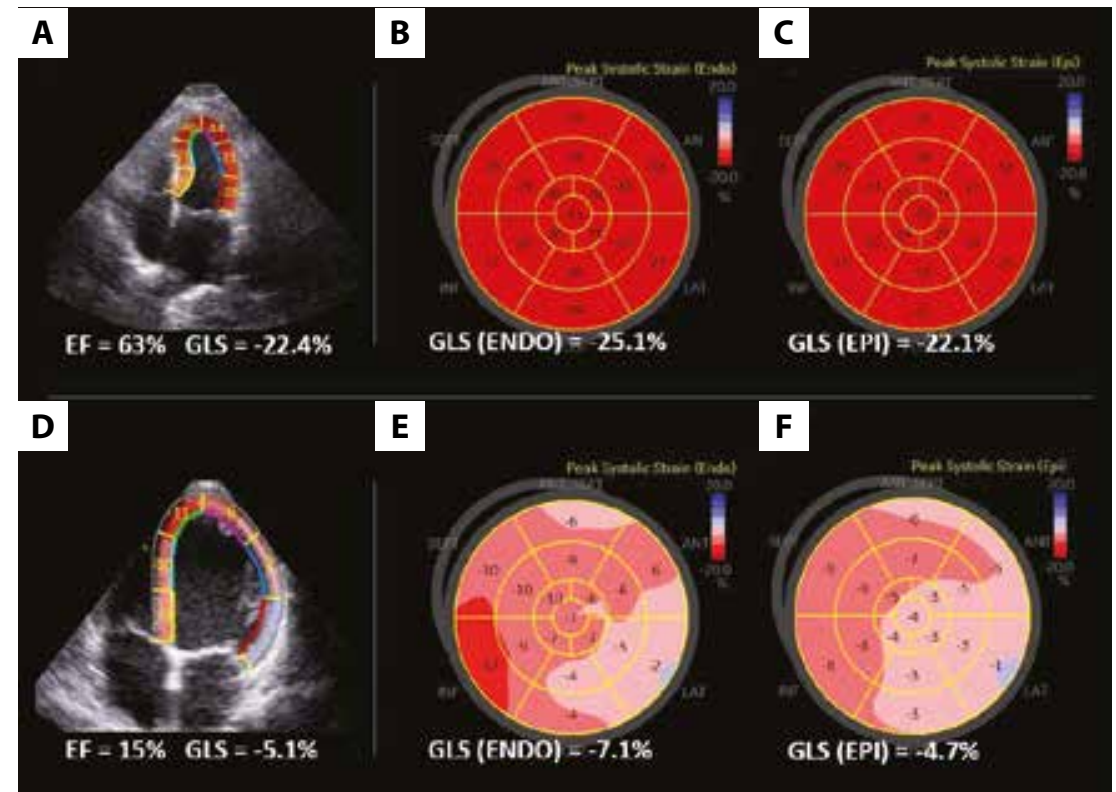

Figure 2. Comparison between the normal (upper panel) and severely impaired myocardial wall deformation expressed by layer-specific strain, in a control subject and a patient from the alcohol group. Upper panels - preserved deformation in a 65-year-old male, arterial hypertension, dyslipidemia, alcohol units per week $=0$. Lower panels - a 63-year-old male with severe impaired deformation, total alcohol dose $=22600$ alcohol units, reported alcohol units per week $=11$, arterial hypertension, dyslipidemia and smoking, but coronary angiography revealed no flow limitations. A. Four-chamber apical view strain regions in a patient with preserved deformation. B. Left ventricular polar map - "bull's eye" of global longitudinal endocardial strain in a patient with preserved deformation. C. Left ventricular polar map - "bull's eye" of global longitudinal epicardial strain in a patient with preserved deformation. D. Four-chamber apical view strain regions in a patient with impaired deformation. E. Left ventricular polar map — "bull's eye" global longitudinal endocardial strain in a patient with impaired deformation. F. Left ventricular polar map — "bull's eye" global longitudinal epicardial strain in a patient with impaired deformation

In the ALC group, the prevalence of abnormal GLS and both layer strains were significantly higher in patients with $\mathrm{EF}<50 \%$ than in those with preserved EF. Among those with reduced $E F$, impaired GLS was observed in 22 patients (88\%) whereas impaired GLSendo and GLSepi were present in 24 (96\%). Among those with preserved $\mathrm{EF}$, impaired GLS was observed in 23 patients $(57 \%)(P=0.02)$, and lowered GLSendo/GLSepi in 26 patients $(65 \%)(P<0.001)$, for both layer strains.
The parameter which in the best manner differentiated ALC and CG was LAVI, which outperformed LVMI and GLS as well as GLSendo whereas, EF <51\% (AUC, 0.770; 95\% confidence interval [95\% Cl], 0.672-0.850) and GLSepi $<15.3 \%$ (AUD, $0.742 ; 95 \% \mathrm{Cl}$ : 0.642-0.826) were not significantly inferior to LAVI. LAVI $>24.5 \mathrm{ml} / \mathrm{m}^{2}$, for the detection of alcohol overusing patients, achieved the highest value among tested parameters (with the area under the curve 
Table 4. The comparison of chosen parameters between groups with and without combined endpoint

\begin{tabular}{|c|c|c|c|}
\hline & Outcome free $(n=54)$ & Combined endpoint $(n=11)$ & $P$-value \\
\hline NT-proBNP, pg/ml, median (IQR) & $281(93-1390)$ & $2192(349-4028)$ & 0.03 \\
\hline $\mathrm{EF}, \%$, median (IQR) & $53(45-58)$ & $37(23-51)$ & 0.01 \\
\hline LVMI, g/m², mean (SD) & $119(43)$ & $164(83)$ & 0.11 \\
\hline LAVI, $\mathrm{ml} / \mathrm{m}^{2}$, median (IQR) & $33(22-64)$ & $33(26-43)$ & 0.77 \\
\hline GLS, \%, mean (SD) & $16(6)$ & $11(5)$ & 0.01 \\
\hline GLSendo, \%, mean (SD) & $18(7)$ & $12(6)$ & 0.01 \\
\hline GLSepi, \%, mean (SD) & $14(6)$ & $9(5)$ & 0.01 \\
\hline
\end{tabular}

Abbreviations: NT-proBNP, N-terminal brain natriuretic peptide, other: see Table 3

[AUC] ROC, 0.848 and with $95 \% \mathrm{Cl}, 0.758-0.915$. This parameter outperformed 3 other tested parameters: LVMI $>118 \mathrm{mg} / \mathrm{m}^{2}$ (AUC, $\left.0.704 ; 95 \% \mathrm{Cl}, 0.599-0.795\right), P=0.02 ; \mathrm{GLS}$ $<17.1 \%$ (AUC, $0.695 ; 95 \% \mathrm{Cl}, 0.592-0.785) ; P=0.01$; GLSendo $<19.9 \%$ (AUC, $0.694 ; 95 \% \mathrm{Cl}, 0.591-0.785$ ); $P=0.04$.

Correlation analysis revealed a very strong relationship between GLSendo, GLSepi and GLS, and GLSendo, GLSepi and EF ( $P<0.001$ for all) (Supplementary material, Figure $S 2$ ). A significant correlation was also observed for GLSendo, GLSepi and NT-proBNP, with a rho value of $0.62(P<0.001)$ for both variables.

The significant positive correlation was observed between FT and GLS (rho $=0.26, P=0.03$ ), as well as a slightly stronger one between FT and GLSendo and GLSepi (rho $=0.31, P=0.01$; rho $=0.30 ; P=0.01$, respectively).

\section{Prognostic value}

Follow-up by direct contact with the patient was achieved in 47 participants (72\%) and life status (dead/alive) was confirmed in all. During follow-up, which lasted for mean 28 (16) months, 11 patients reached the combined endpoint. Ten hospitalizations were recorded and assessed as HF exacerbation, according to the patients' reports. In eight cases, hospitalization was related to peripheral edema and dyspnea and in two cases to symptomatic arrhythmias. Death was reported by the patient's relatives as of cardiac origin.

A comparison between outcome-free patients and the group with combined endpoint found the group with worse prognosis to have significantly higher NT-proBNP, as well as lower EF and lower absolute values for all strain parameters (Table 4).

The ROC curve analysis found NT-proBNP with a cut-off value $>2058 \mathrm{pg} / \mathrm{ml}$ to have $0.733 \mathrm{AUC}(95 \% \mathrm{Cl}, 0.582-0.852)$ for predicting the combined endpoint. The respective cut-off and ROC AUC values for the tested variables for detecting the combined endpoint were as follows: $\mathrm{EF}$ $\leq 26 \%$ (AUC, 0.739; 95\% Cl, 0.611-0.843), GLS <18 (AUC, $0.751 ; 95 \% \mathrm{Cl}, 0.624-0.853$ ), GLSepi <15\% (AUC, 0.750; 95\% $\mathrm{Cl}, 0.623-0.852$ ), and GLSendo <19\% (AUC, $0.747 ; 95 \% \mathrm{Cl}$, $0.620-0.850)$.

Adding EF to NT-proBNP increased the value of AUC to 0.769 (95\% Cl: $0.621-0.880)$ but this difference was not statistically significant $(P=0.40)$. Similarly, adding strain values slightly increased AUC ROC to $0.796(95 \% \mathrm{Cl}, 0.651-$ $0.900)$; however, this value was not statistically significant ( $P=0.24$ vs NT-proBNP alone and $P=0.45$ vs NT-proBNP + EF) (Supplementary material, Figure S3). Kaplan-Meier survival curves were constructed for patients with GLSepi $<15 \%$ and GLSendo <19\% (Supplementary material, Figure S4).

\section{DISCUSSION}

\section{Alcohol influence on cardiac function}

Park et al. [10] report that LV diastolic impairment was associated with a higher alcohol consumption: diastolic dysfunction was observed in 1758 individuals (32\%) in a"heavy drinking group" consuming 30-60 g of pure alcohol per day (21-42 AUW), compared to 853 (16\%) in an "occasional drinking group" consuming $<10 \mathrm{~g}$ of pure alcohol per day ( $<7 \mathrm{AUW}) ; P<0.001$. The median alcohol dose consumed by the heavy drinkers was similar to the median (IQR) amount observed in our present study, i.e., 30 AUW (12-51). In addition, diastolic dysfunction was detected in 26 subjects (40\%) in the ALC in our present study. Moreover, Park et al. [10] report higher mean LVMI 78 (14) $\mathrm{g} / \mathrm{m}^{2}$ in the "heavy drinking group"than the "occasional drinking group" 70 (14) $\mathrm{g} / \mathrm{m}^{2}, P<0.001$. In the present study, higher median (IQR) LVMI was found in the ALC group, i.e., 119 (91-155), than controls, i.e. $93(75-110) \mathrm{g} / \mathrm{m}^{2} ; P=0.002$; in addition, the ALC group also demonstrated thicker IVS and PW.

Daily alcohol consumption greater than $80 \mathrm{~g}$ of pure alcohol (equal $>56$ AUW) for $\geq 5$ years has been associated with the development of alcohol cardiomyopathy $[2,3,11]$. However, our findings also indicate systolic and diastolic dysfunction in the ALC group, who were consuming slightly above half the dose of alcohol, i.e., 30 AUW (12-51), but over a longer mean period of time, i.e. - mean (SD) 16 years (9). In our ALC group, 89\% of patients reported symptoms corresponding to New York Heart Association (NYHA) $\geq 1$ class. This clinical presentation may be partially related to liver disease or malnutrition caused by alcohol abuse [12].

\section{Layer-specific strain in cardiac damage}

LV function can be more precisely evaluated in different clinical settings, e.g., in ischemia detection, by including deformation parameters [13]. Layer-specific strain was found to be a marker of cardiac muscle dysfunction asso- 
ciated with arterial hypertension and LV hypertrophy [14, 15]. Similarly, absolute GLS values have been found to be lower in toxic cardiomyopathies [16-20].

A study of 44 patients with hypertrophic obstructive cardiomyopathy found them to have a mean (SD) IVS thickness of $18.9 \mathrm{~mm}$ (7.5) and mean (SD) PW thickness of $11.83 \mathrm{~mm}$ (3.42), compared to otherwise healthy controls with a mean (SD) IVS thickness of $8.57 \mathrm{~mm}$ (1.16) and mean (SD) PW thickness of $9.14 \mathrm{~mm}$ (1.42). Absolute layer-specific strain values were also found to be lower in the cardiomyopathy group: GLSendo was $15.31 \%$ (5.55\%) compared to $22.04 \%$ (2.88\%); $P<0.01$, and GLSepi was $11.78 \%$ (4.28\%) compared to $17.81 \%(2.81 \%)$ in controls; $P<0.01$ (14).

In the present study, median (IQR) GLSendo absolute values were $19 \%(12 \%-23 \%)$ in ALC vs $24 \%(21 \%-27 \%)$ in CG $(P=0.007)$, and absolute GLSepi was $14 \%(9 \%-18 \%)$ vs $19 \%(16 \%-20 \%)(P=0.007)$. The presence of a better-preserved layer strain in our ALC group might be partly related to the relatively higher implementation of hypertension therapy. This may exert a protective effect on LV function: mean (SD) EF was found to increase from 34\% (8\%) to 39\% $(8 \%)(\mathrm{P}<0.001)$ during six months of observation in HF patients with spironolactone added to the therapy [21]. The literature concerning the layer-specific strain associated with chronic alcohol exposure is rather sparse, and it is difficult to obtain accurate information about alcoholism severity, often due to under-rated self-reported alcohol consumption [22]. Nevertheless, in a study of 199 healthy patients, mean age 44 (5) years, $71 \%$ males, in the acute phase of alcohol toxicity, the mean (SD) alcohol level in blood sample six hours after alcohol ingestion was 1.2 (0.3) $\mathrm{g} / \mathrm{L}$. The mean (SD) GLSendo during alcohol exposure was $27 \%(4 \%)$, significantly lower than the value taken after four weeks of strict alcohol abstinence, i.e. 33\% (3\%) $(P=0.002)$. Blood pressure, EF, heart rate, and other classical echocardiographic parameters did not show any difference between the two examinations [23].

\section{Prognostic value of layer-specific strain}

Deformation parameters have demonstrated diagnostic and prognostic utility in a wide range of cardiac and non-cardiac conditions, such as ischemic heart disease [13], aortic stenosis [24], diabetes type 2 [25], acute hyperglycemia [26] or pre-eclampsia [27]. Both GLS and layer-specific strains (GLSendo, GLSepi) have been found to have predictive significance for adverse outcomes following acute coronary syndrome [28]. Skaarup et al. [28] observed that global longitudinal strain is independently associated with the combined outcome (HF or cardiovascular symptom development) for lowered GLSendo (HR, 1.19 [1.10-1.28]; $P<0.001)$ and GLSepi (HR, 1.26 [1.15-1.39]; $P<0.001)$. In our study, univariate analysis found elevated NT-proBNP $>2058 \mathrm{pg} / \mathrm{ml}$ decreased EF $\leq 26 \%$ and longitudinal strain (GLS $<18 \%$; GLSepi $<15.1 \%$; GLSendo $<19 \%$ ) were related to a worse prognosis in the ALC group. Logistic regression analysis found that all tested parameters similarly discerned the outcomes which were not significantly improved in models based on two or three variables; this was probably due to the insufficient number of observed endpoints.

\section{Study limitations}

The study does have some limitations. The main one is that it only includes a relatively small number of examined patients. This is partially related to the difficulties of cooperating with addicted people and lower compliance in direct follow-up. However, the study also places an emphasis on 2D longitudinal strain and LV assessment. Further information could be gathered by including information gathered by 3D strain variables, rotation and torsion analysis, as well as deformation of right ventricle. In addition, it is difficult to objectively quantify self-reported alcohol doses, which may be underrated. Furthermore, the duration of the follow-up was relatively short, and only a limited number of combined endpoints were used, with cardiac origin established only by phone interview. Finally, differences in hypertension treatment were used between groups; this could influence cardiac function and prognosis outcomes, potentially improving natural history of HF in the affected group.

\section{CONCLUSIONS}

Patients reporting alcohol overuse, with a mean (SD) 16 (9) year consumption of median (IQR) 4 (2-7) alcohol units per day, demonstrated the features of LV hypertrophy, left atrial enlargement, and LV diastolic and systolic dysfunction.

Regarding myocardial deformation, GLS and layer strain showed lower absolute values in alcohol overusers as compared to controls; both were associated with a more frequent occurrence of combined endpoint: a higher clinical risk of death or cardiovascular hospitalization for GLSendo $<19 \%$ and GLSepi < $15 \%$. Nevertheless, similarly to volumetric assessment of atrium (LAVI), quantitative assessment of deformation (GLS, GLSendo and GLSepi) did not add any significant predictive value to models based on EF and NT-proBNP.

Moreover, the duration of abstinence from the last drinking episode correlated positively with the absolute value of longitudinal strain; this indicates the value of quantitative strain data for assessing functional recovery based on abstinence and pharmacological treatment.

\section{Supplementary material}

Supplementary material is available at https://journals. viamedica.pl/kardiologia_polska.

\section{Article information}

Conflict of interest: None declared.

Open access: This article is available in open access under Creative Common Attribution-Non-Commercial-No Derivatives 4.0 International (CC BY-NC-ND 4.0) license, allowing to download articles and share them with others as long as they credit the authors and the publisher, but without permission to change them in any way or use 
them commercially. For commercial use, please contact the journal office at kardiologiapolska@ptkardio.pl.

How to cite: Hamala P, Kasprzak JD, Bińkowska A, et al. The impact of chronic alcohol overuse on cardiac function and prognosis: layer-specific longitudinal strain and mid-term outcome analysis. Kardiol Pol. 2021; 79(7-8): 781-788, doi: 10.33963/KP.15986.

\section{REFERENCES}

1. World Health Organization. Global status report on alcohol and health 2018. https://apps.who.int/iris/handle/10665/274603 (December 28, 2018).

2. Guzzo-Merello G, Segovia J, Dominguez F, et al. Natural history and prognostic factors in alcoholic cardiomyopathy. JACC Heart Fail. 2015; 3(1): 78-86, doi: 10.1016/j.jchf.2014.07.014, indexed in Pubmed: 25458176.

3. Guzzo-Merello G, Cobo-Marcos M, Gallego-Delgado M, et al. Alcoholic cardiomyopathy. World J Cardiol. 2014; 6(8): 771-781, doi: 10.4330/wjc. v6.i8.771, indexed in Pubmed: 25228956.

4. Shi J, Pan C, Kong D, et al. Left ventricular longitudinal and circumferential layer-specific myocardial strains and their determinants in healthy subjects. Echocardiography. 2016; 33(4): 510-518, doi: 10.1111/echo.13132, indexed in Pubmed: 26661049.

5. Sugimoto T, Robinet S, Dulgheru R, et al. NORRE Study. Echocardiographic reference ranges for normal left atrial function parameters: results from the EACVI NORRE study. Eur Heart J Cardiovasc Imaging. 2018; 19(6): 630-638, doi: 10.1093/ehjci/jey018, indexed in Pubmed: 29529180.

6. Mor-Avi V, Lang RM, Badano LP, et al.Current and evolving echocardiographic techniques for the quantitative evaluation of cardiac mechanics: ASE/EAE consensus statement on methodology and indications endorsed by the Japanese Society of Echocardiography. J Am Soc Echocardiogr. 2011;24(3): 277-313, doi: 10.1016/j.echo.2011.01.015, indexed in Pubmed: 21338865.

7. Sugimoto T, Dulgheru R, Bernard A, et al. Echocardiographic reference ranges for normal left ventricular $2 \mathrm{D}$ strain: results from the $\mathrm{EACVI}$ NORRE study. Eur Heart J Cardiovasc Imaging. 2017; 18(8): 833-840, doi: 10.1093/ehjci/jex140, indexed in Pubmed: 28637227.

8. Tsugu T, Postolache A, Dulgheru R, et al. Echocardiographic reference ranges for normal left ventricular layer-specific strain: results from the EACVI NORRE study. Eur Heart J Cardiovasc Imaging. 2020; 21(8):896-905, doi: 10.1093/ehjci/jeaa050, indexed in Pubmed: 32259844.

9. Manganaro R, Marchetta S, Dulgheru R, et al. Echocardiographic reference ranges for normal non-invasive myocardial work indices: results from the EACVI NORRE study. Eur Heart J Cardiovasc Imaging. 2019; 20(5): 582-590, doi: 10.1093/ehjci/jey188, indexed in Pubmed: 30590562.

10. Park SK, Moon K, Ryoo JH, et al. The association between alcohol consumption and left ventricular diastolic function and geometry change in general Korean population. Eur Heart J Cardiovasc Imaging. 2018; 19(3): 271-278, doi: 10.1093/ehjci/jex091, indexed in Pubmed: 28459993.

11. Ware JS, Amor-Salamanca A, Tayal U, et al. Genetic etiology for alcohol-induced cardiac toxicity. J Am Coll Cardiol. 2018; 71(20): 2293-2302, doi: 10.1016/j.jacc.2018.03.462, indexed in Pubmed: 29773157.

12. Kamran U, Towey J, Khanna A, et al. Nutrition in alcohol-related liver disease: Physiopathology and management. World J Gastroenterol. 2020; 26(22): 2916-2930, doi: 10.3748/wjg.v26.i22.2916, indexed in Pubmed: 32587439.

13. Mielczarek A, Kasprzak JD, Lipiec $P$, et al. Quantitative assessment of the rotation and twist of the left ventricle during dobutamine stress echocardiography: a comparison of patients with and without significant coronary artery disease. Kardiol Pol. 2019; 77(7-8): 696-702, doi: 10.33963/KP.14870, indexed in Pubmed: 31215525.

14. Kim SA, Park SM, Kim MN, et al. Assessment of left ventricular function by layer-specific strain and its relationship to structural remodelling in patients with hypertension. Can J Cardiol. 2016; 32(2): 211-216, doi: 10.1016/j.cjca.2015.04.025, indexed in Pubmed: 26255215.
15. Zhang J, Zhu L, Jiang $X$, et al. Layer-specific strain analysis of left ventricular myocardium after alcohol septal ablation for hypertrophic obstructive cardiomyopathy. Medicine (Baltimore). 2018; 97(45): e13083, doi: 10.1097/MD.0000000000013083, indexed in Pubmed: 30407309.

16. Kucuk M, Oncel CR, Belgi Yıldırım A, et al. Evaluation of subclinical left ventricular systolic dysfunction in chronic asymptomatic alcoholics by speckle tracking echocardiography. Biomed Res Int. 2017; 2017:6582568, doi: 10.1155/2017/6582568, indexed in Pubmed: 28466016.

17. Hamala $P$, Kasprzak JD, Lipiec $P$, et al. P4134 impaired left ventricular systolic function in alcohol abusers expressed by both 3D calculated ejection fraction and longitudinal strain by AFI. Eur Heart J. 2019; 40(Suppl 1): ehz745.0706, doi: 10.1093/eurheartj/ehz745.0706.

18. Yang $\mathrm{H}$, Wright $L$, Negishi $\mathrm{T}$, et al. Research to practice: assessment of left ventricular global longitudinal strain for surveillance of cancer chemotherapeutic-related cardiac dysfunction. JACC Cardiovasc Imaging. 2018; 11(8): 1196-1201, doi: 10.1016/j.jcmg.2018.07.005, indexed in Pubmed: 30092974.

19. de Almeida Gripp E, de Oliveira GE, Feijó LA, et al. Global longitudinal strain accuracy for cardiotoxicity prediction in a cohort of breast cancer patients during anthracycline and/or trastuzumab treatment. Arq Bras Cardiol. 2018; 110(2): 140-150, doi: 10.5935/abc.20180021, indexed in Pubmed: 29561992.

20. Edwards NC, Hirth A, Ferro CJ, et al. Subclinical abnormalities of left ventricular myocardial deformation in early-stage chronic kidney disease: the precursor of uremic cardiomyopathy? J Am Soc Echocardiogr. 2008; 21(12): 1293-1298, doi: 10.1016/j.echo.2008.09.013, indexed in Pubmed: 19041571.

21. Vizzardi E, Sciatti E, Bonadei l, et al. Effects of spironolactone on ventricular-arterial coupling in patients with chronic systolic heart failure and mild symptoms. Clin Res Cardiol. 2015; 104(12): 1078-1087, doi: 10.1007/s00392-015-0877-5, indexed in Pubmed: 26058790.

22. Kozela M, Doryńska A, Bobak M, et al. Alcohol use disorder increases the risk of nonfatal and fatal cardiovascular disease: an 11-year follow-up of a Polish population-based cohort. The HAPIEE study. Pol Arch Intern Med. 2020; 130(11): 960-966, doi: 10.20452/pamw.15616, indexed in Pubmed: 32969635

23. Schröder J, Hamada S, Altiok E, et al. Detection of acute changes in left ventricular function by myocardial deformation analysis after excessive alcohol ingestion. J Am Soc Echocardiogr. 2017; 30(3): 235-243.e1, doi: 10.1016/j.echo.2016.12.009, indexed in Pubmed: 28259304.

24. Ilardi F, Marchetta S, Martinez C, et al. Impact of aortic stenosis on layer-specific longitudinal strain: relationship with symptoms and outcome. Eur Heart J Cardiovasc Imaging. 2020; 21(4): 408-416, doi: 10.1093/ehjci/jez215, indexed in Pubmed: 31504364.

25. Wierzbowska-Drabik K, Trzos E, Kurpesa M, et al. Diabetes as an independent predictor of left ventricular longitudinal strain reduction at rest and during dobutamine stress test in patients with significant coronary artery disease. Eur Heart J Cardiovasc Imaging. 2018; 19(11): 1276-1286, doi: 10.1093/ehjci/jex315, indexed in Pubmed: 29236974.

26. Bogdanović J, Ašanin M, Krljanac G, et al. Impact of acute hyperglycemia on layer-specific left ventricular strain in asymptomatic diabetic patients: an analysis based on two-dimensional speckle tracking echocardiography. Cardiovasc Diabetol. 2019; 18(1): 68, doi: 10.1186/s12933-019-0876-3, indexed in Pubmed: 31159858.

27. Cong J, Lee $Y, F u X$, et al. Quantitative evaluation of longitudinal strain in layer-specific myocardium in patients with preeclampsia. Int J Cardiovasc Imaging. 2018;34(2): 193-200, doi: 10.1007/s10554-017-1220-1, indexed in Pubmed: 28776114.

28. Skaarup KG, Iversen A, Jørgensen PG, et al. Association between layer-specific global longitudinal strain and adverse outcomes following acute coronary syndrome. Eur Heart J Cardiovasc Imaging. 2018; 19(12): 1334-1342, doi: 10.1093/ehjci/jey004, indexed in Pubmed: 29617974. 